\title{
S'alimenter malgré le froid, la distance et le reste : l'émergence de stratégies favorables à la résilience alimentaire en Jamésie (nord du Québec)
}

\author{
France Desjardins ${ }^{a}$, Pierre-André Tremblayb
}

RÉSUMÉ. Cet article s'intéresse à la résilience démontrée par les acteurs du système alimentaire de la Jamésie, dans le nord du Québec, en se basant sur les stratégies déployées par les personnes qui se trouvent à l'extrémité de la chaîne de consommation, soit les «mangeurs». Dans cette région et ce système alimentaire, nous retrouvons des acteurs économiques liés au système alimentaire (producteurs, transformateurs, distributeurs et transporteurs), mais aussi des organisations communautaires et des instances politico-administratives. L'analyse repose sur le fait que les mangeurs possèdent des caractéristiques individuelles et sociales qui organisent, permettent et contraignent leurs stratégies. Ces résultats d'une recherche réalisée en 2020 ont été intégrés à une réflexion systémique relative à l'interdépendance des acteurs afin de proposer des recommandations visant à développer la participation et la coordination des diverses parties prenantes.

\begin{abstract}
This article looks into the resilience demonstrated by actors in the Jamésie food system, in the north of Quebec, based on the strategies of the "eaters", who are at the end of the consumption chain. In this region and in this food system, we find economic actors linked to the food system (producers, processors, distributors and shippers), but also community organizations and politico-administrative bodies. The analysis is based on the fact that eaters have individual and social characteristics that organize, enable and constrain their strategies. These results of a research carried out in 2020 were integrated into a systemic reflection on the interdependence of actors, in order to propose recommendations aimed at developing the participation and coordination of the various stakeholders.
\end{abstract}

\section{Introduction}

Le Québec est signataire de la Déclaration de Rome sur la sécurité alimentaire mondiale (FAO, 1996) et l'a intégrée à différentes lois. À titre d'exemple, la Loi visant à lutter contre la pawvreté et l'exclusion sociale adoptée en 2002 par le gouvernement du Québec propose de «favoriser, pour les personnes et les familles en situation de pauvreté, l'accès, en toute dignité, à un approvisionnement alimentaire suffisant et nutritif, à un coût raisonnable, de même qu'à une information simple et fiable qui leur permette de faire des choix alimentaires éclairés» (Québec, 2002, paragr. 9[4]). Puis, en 2008, le ministère de la Santé et des Services sociaux du Québec a publié le Cadre de référence en matière de sécurité alimentaire (Pageau, 2008) pour s'assurer de l'application de la sécurité alimentaire.

Malgré tout, près de $11,1 \%$ des ménages québécois vivaient, en 2017-2018, une situation d'insécurité alimentaire allant de modérée à grave. En outre, ces ménages n'avaient pas accès à des aliments ou ne pouvaient pas combler leurs besoins en matière d'alimentation (Statistique Canada, 2017, 2018). Plus récemment, les Banques alimentaires du Québec (2020) donnaient des denrées à environ 500000 personnes chaque mois, un nombre que la pandémie de COVID-19 a fait croitre de plus de $40 \%$.

\footnotetext{
${ }^{a}$ Chargée de cours, membre du Laboratoire de recherche et d'intervention en gouvernance des organisations (LARIGO), membre associé du Laboratoire d'études multidisciplinaires en gestion de projet (LemGP), Université du Québec à Chicoutimi

${ }^{\mathrm{b}}$ Professeur associé au Département des sciences humaines et sociales, membre du Centre de recherche sur les innovations sociales (CRISES) et du Groupe de recherche et d'intervention régionales, Université du Québec à Chicoutimi
} 
L'insécurité alimentaire, la sécurité alimentaire et les acteurs du système alimentaire ont été étudiés pour de nombreux territoires; les milieux urbains, ruraux, nordiques (surtout avec les Premières Nations), tropicaux et d'autres sont couramment étudiés. Toutefois, au Québec, le secteur de la Jamésie a suscité peu d'intérêt de la part des chercheurs. En 2019, le Centre régional de santé et de services sociaux (CRSSS) de la Baie-James, qui est responsable de l'application du Cadre de référence en matière de sécurité alimentaire pour la population de son territoire, s'est questionné sur les enjeux, les défis et les besoins alimentaires des «mangeurs». L'entente avec cette institution pour réaliser une recherche scientifique vise à documenter la situation de la sécurité et de l'insécurité alimentaires des mangeurs résidant sur le territoire de la Jamésie dans une perspective d'amélioration de la situation collective ${ }^{1}$.

Le recours à une vision systémique de la sécurité alimentaire pourrait apporter un éclairage à la notion de résilience alimentaire à différentes échelles (individu, ménage, famille, communauté), comme le suggèrent des acteurs du développement (Vonthron et collab., 2016; UNFPA, 2015; USAID, 2012; DFID, 2011). Cette vision met de l'avant la créativité et l'esprit d'initiative des résidents des collectivités, qu'on ne peut réduire au rôle passif de consommateurs, ni à celui de porteurs de contraintes. Avec cette perspective de développement, des pistes d'action liées à l'innovation sociale peuvent être soumises.

Cet article s'intéresse à la résilience démontrée par les acteurs du système alimentaire de la Jamésie en se basant sur les stratégies qu'ils et elles déploient. Ces stratégies nous permettent d'approfondir les notions de résilience alimentaire et d'innovation.

Les principales questions auxquelles notre recherche tente de trouver des réponses sont :
Quelles sont les stratégies déployées par les informateurs pour atteindre la sécurité à l'intérieur du système alimentaire? Comment la collectivité, incluant les multiples acteurs du système alimentaire, peut-elle contribuer ou non à la mise sur pied et à la reproduction de ces stratégies?

Afin de répondre à ces questions, nous exposerons d'abord à grands traits le contexte de la Jamésie. Notre cadre théorique présentera les principales notions permettant d'aborder la (in)sécurité alimentaire. Les questions et la méthodologie de recherche suivront. La présentation des résultats et leur discussion concluront l'article.

\section{Contexte géographique et sociologique}

La région de la Baie-James se situe dans le nordouest de la province du Québec, au Canada, entre les $49^{\mathrm{e}}$ et $55^{\mathrm{e}}$ parallèles (voir Figure 1). Faisant partie de la région administrative du Nord-du-Québec, elle a une superficie de $339698 \mathrm{~km}^{2}$ et la distance de l'est à l'ouest totalise $640 \mathrm{~km}$. Elle représente $22 \%$ de tout le territoire québécois (CRSSS de la Baie-James, 2020).

La Jamésie regroupe quatre villes (Chibougamau, Lebel-sur-Quévillon, Matagami et Chapais) ainsi que trois localités ${ }^{2}$ (Radisson, Villebois et Valcanton (fusion entre Val-Paradis et Beaucanton)). Elle compte une population allochtone de 13575 personnes (Statistique Canada, 2016). Une population autochtone crie de 17141 personnes habite également ce territoire. Au total, la population est donc de 30716 personnes, soit 11,5 personnes au kilomètre carré. Puisque les Cris possèdent leur propre réseau de santé et de services sociaux (CRSSS de la Baie-James, 2020), ils n’ont pas été inclus dans notre recherche, qui s'est concentrée sur les populations qui n'appartiennent pas aux Premières Nations. 


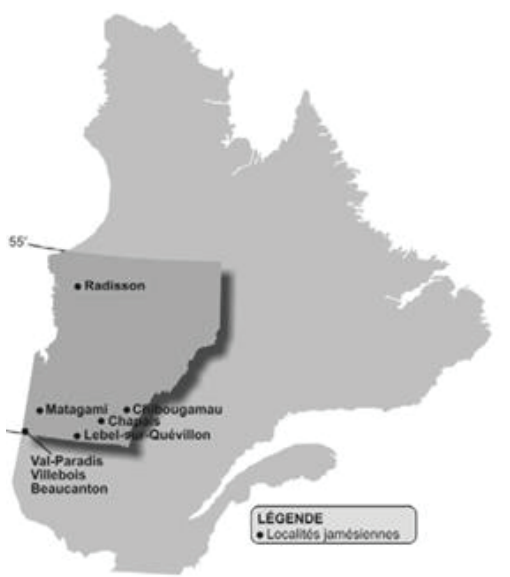

Figure 1 - Localisation de la Jamésie (villes et localités) Source : CRSSS de la Baie-James (2020)

La plupart des villes/localités connaissent une baisse de leur population, bien qu'à des rythmes variables et fluctuant au gré des conditions socioéconomiques. L'âge médian se situe généralement entre 40 et 45 ans. En 2015, plus du tiers des ménages jamésiens vivait avec moins de 50000 \$ par année après impôt.

Dans certaines villes/localités, près de la moitié de la population âgée de 25 à 64 ans ne possède aucun certificat, diplôme ni grade scolaire, mais, en général, cette faible scolarité touche de $6 \%$ à $26 \%$ de la population jamésienne.

L'économie régionale est essentiellement extractive, donc repose sur l'exploitation des ressources naturelles. Les principaux secteurs sont la gestion de l'hydroélectricité (centrales et barrages), l'exploitation des mines, de la forêt et des ressources fauniques ainsi que le tourisme. Le secteur agroalimentaire est très peu développé, mais présente une activité économique relativement stable. Quelques agriculteurs produisent des pommes de terre, des bleuets, des tomates ou font l'élevage de bovins, essentiellement dans la partie ouest de la région.

Cette situation excentrique, les conditions climatiques, la grandeur du territoire peu peuplé et les caractéristiques de l'économie régionale donnent un sens particulier à la notion de sécurité alimentaire et obligent à ne pas la réduire à la seule production locale de nourriture. La sécurisation implique une transformation des habitudes des personnes, c'est-à-dire un accent sur leur agentivité - ce que Latour (2015) appelle les «puissances d'agir»-, qui accorde une grande importance à l'empowerment individuel et collectif. De plus, comme le suggèrent Fallot et ses collègues (2019), il y a nécessité d'analyser les limites des capacités des ménages pour identifier les leviers structurels, étatiques et publics qui pourraient être déployés par ces acteurs.

\section{Cadre théorique}

De notre point de vue, les stratégies alimentaires des acteurs sont liées au système alimentaire, que Malassis (1994) entend comme «la façon dont les hommes [sic] s'organisent dans l'espace et dans le temps pour obtenir et consommer leur nourriture» (p. 7). Selon lui, les acteurs du système alimentaire peuvent être les fournisseurs de matériaux, les agriculteurs, les transformateurs, les distributeurs, les chauffeurs, les chercheurs, les formateurs, les employés des services financiers, etc. Rastoin et Ghersi (2010) insistent avec justesse sur l'importance de l'interdépendance entre ces divers acteurs économiques ainsi que sur leur but : la satisfaction des besoins alimentaires de certains consommateurs. Le constat global est qu'un grand nombre d'acteurs sont mobilisés de manière interdépendante pour réaliser une ou plusieurs actions de façon linéaire afin d'apporter aux consommateurs ciblés un produit de consommation qui a un prix, c'est-à-dire une valeur économique.

Les éléments clés de cet écosystème structurel sont que les mangeurs possèdent des caractéristiques individuelles et sociales. Ces derniers vivent dans une région géographique ayant des particularités économiques, culturelles, technologiques, organisationnelles et structurelles. Au sein de cette région, nous retrouvons entre autres des acteurs économiques liés au système alimentaire tels que des producteurs, des transformateurs, des distributeurs, des transporteurs 
ainsi que des associations et des instances politicoadministratives. Le mot «système» est utilisé parce qu'il désigne un ensemble d'éléments (moyens, méthodes, règles) utilisés par les différents acteurs visant un but défini (Mélèze, 1972), celui-ci étant de nourrir les citoyens de la région. La figure 2 illustre les acteurs présents au sein du système alimentaire.

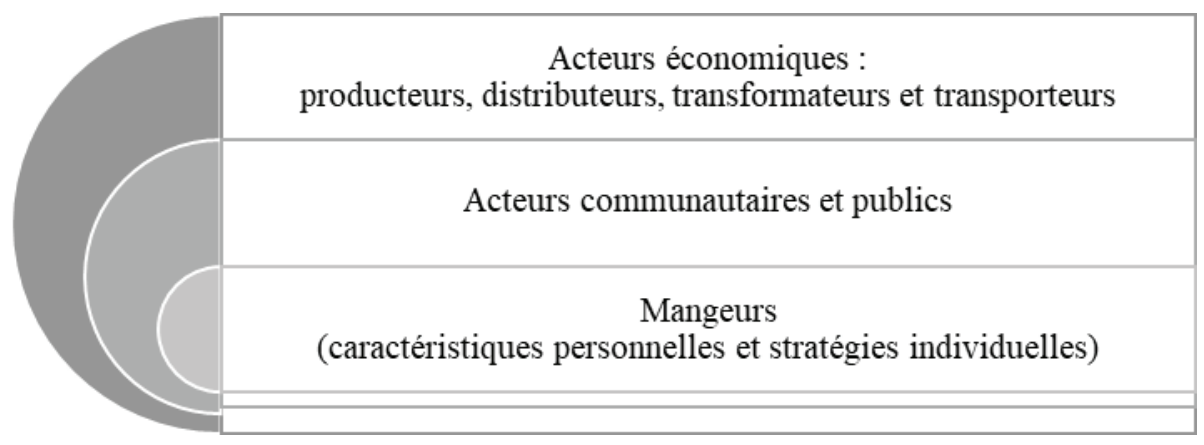

Figure 2-Cadre systémique de référence du système alimentaire de la Jamésie

Le cercle central de la figure 2 représente les mangeurs, qui sont notre préoccupation majeure. Notons que ces mangeurs sont, pour nous, les résidents allochtones sur le territoire, peu importe leur revenu. Toutefois, de manière logique, si ceux qui disposent d'un faible revenu sont capables de jouir d'une sécurité alimentaire, tous le pourront.

Entourant ces individus, on retrouve les acteurs économiques, dont les producteurs, les distributeurs, les transformateurs et les transporteurs, c'est-à-dire les acteurs qui travaillent à se rendre physiquement au lieu d'alimentation. Puis, on retrouve les acteurs communautaires et publics.

Tous ces éléments permettront de répondre à l'objectif de cet article: comprendre la manière dont les mangeurs parviennent à combler leurs besoins alimentaires. Nous privilégierons en particulier les liens entre ces mangeurs et l'implication (ou, le cas échéant, l'absence d'implication) des divers acteurs du système alimentaire que sont les collectivités et les acteurs communautaires, publics, étatiques et économiques.

Les ressources financières constituent un élément fondamental de la situation des mangeurs, car elles peuvent avoir un impact sur leurs choix et leurs stratégies alimentaires. Cette notion implique que les personnes à faible revenu peuvent éprouver des difficultés à combler leurs besoins alimentaires, malgré leur connaissance d'une saine alimentation (Damon, 2012).

La conséquence est importante : pour se nourrir, ces personnes devront utiliser toutes sortes de moyens de stratégies - tels que le recours aux associations ou à des activités collectives (p. ex., les jardins communautaires ou les cuisines collectives). De façon généralement concurrente, ces personnes auront aussi recours à l'aide alimentaire d'urgence (don). Or, cela ne se fait souvent pas sans mal : selon Dupéré et ses collègues (2014), les utilisateurs des ressources communautaires en matière de sécurité alimentaire disent éprouver de la honte, être blessés dans leur orgueil et craindre d'être étiquetés. D'autres stratégies peuvent être utilisées, soit le troc ou le soutien familial (Côté, 2007). Dans certaines régions du Québec, la chasse ainsi que la pêche (Bergeron et collab., 2015) sont aussi des avenues possibles. Ainsi, nous pouvons définir qu'une stratégie regroupe un ensemble d'objectifs et de choix qui orientent les activités réalisées par les mangeurs.

Tout comme le gouvernement du Québec, l'instance administrative responsable de la santé publique avec laquelle nous avons collaboré se préoccupe de la possibilité d'augmentation de l'insécurité alimentaire et favorise une approche basée sur la notion de résilience. Ce terme signifie «la capacité pour un ménage, un territoire ou une autre entité faisant partie du système de faire face à des perturbations : les absorber, s'adapter ou se réorganiser» (Folke et collab., 2010, cités dans Fallot et collab., 2019). Dans une telle perspective, l'augmentation de l'insécurité pourrait résulter des difficultés répétitives vécues par différents maillons du système. Ainsi, lorsqu'un ou plusieurs acteurs s'impliquent à l'élaboration de stratégies propices à la réorganisation du système alimentaire, nous pouvons parler d'une innovation, comme le suggère Mulgan (2006) $)^{3}$. 
De manière globale, la sécurité alimentaire peut donc être décrite comme un processus de coordination et d'intégration des différents acteurs du système alimentaire (mangeurs, collectivité ainsi qu'acteurs communautaires, publics, étatiques et économiques) qui vise le déploiement d'une autonomie alimentaire accessible à tous, en tout temps.

Cette proposition fait également référence à la résilience alimentaire, qui renvoie à la capacité du système alimentaire et de ses constituants de garantir la sécurité alimentaire des citoyens en tout temps, malgré des événements perturbateurs de l'environnement général (Tendall et collab., 2015). Cette capacité des acteurs présents au sein du système alimentaire à vouloir combler les besoins des mangeurs peut contribuer à l'émergence de nouvelles stratégies (Touzard et Fournier, 2014).

\section{Méthodologie}

Ces éléments permettent de poser en termes plus concrets les questions de recherche présentées plus haut. L'exploration de la réalité empirique a été faite au moyen d'une étude de cas multisite (Miles et Huberman, 1994) qualitative, qui met l'accent sur la compréhension des phénomènes pour les acteurs (Creswell et Poth, 2018). Les informations ont été recueillies auprès d'un nombre de personnes nécessairement réduit $(\mathrm{n}=40)$ afin de garder la recherche à l'intérieur de limites raisonnables.

Les personnes rencontrées ont été classées en trois catégories basées sur leur place dans le système alimentaire : 1) les mangeurs (certains en situation d'insécurité alimentaire, d'autres non); 2) les intervenants et intervenantes publics ou communautaires; 3) les personnes participant à la chaîne de production et de distribution alimentaire (p. ex., commerçants, agriculteurs et consultants), bien que peu de distributeurs aient pu être rejoints. Les sites étaient les villes et localités allochtones de la Jamésie.

Parmi les mangeurs, 16 femmes et 8 hommes ont participé à la recherche. Ils sont de tous les âges (de 20 à 80 ans) et de toutes les conditions financières (de bénéficiaires de la sécurité du revenu jusqu'aux hauts salariés). Deux personnes ont des handicaps physiques et une personne est issue de l'immigration internationale. La moitié des participants ont des enfants et huit sont célibataires. Certains mangeurs travaillent dans des commerces alimentaires, des organisations publiques ou des organismes communautaires.

Pour ce qui est des acteurs économiques, publics et communautaires, certains ont été choisis pour leur participation à une entreprise et d'autres pour leur implication dans des projets.

Des entretiens semi-structurés, d'une durée de 30 à 75 minutes, ont permis d'aborder leurs opinions et leurs expériences concernant la manière dont ils parviennent à combler leurs besoins alimentaires.

\section{Résultats}

Dans la présentation des données, nous avons classé dans les trois catégories indiquées ci-dessus les acteurs organisationnels pertinents selon les stratégies qu'ils déploient en matière de sécurité alimentaire. Au cœur de leurs stratégies, comme nous le verrons, se retrouvent les enjeux et les occasions de développement.

\subsection{Les acteurs économiques}

\section{Les producteurs}

En matière de production alimentaire, la Jamésie compte une dizaine d'établissements agricoles ${ }^{4}$. Ces établissements font de l'élevage de bovins, tandis que l'éventail de la production végétale comprend les céréales, les fourrages, le bleuet en milieu forestier et la pomme de terre. Pour certains producteurs agricoles rencontrés, le développement de l'agriculture et sa rentabilité représentent des défis et des enjeux liés au climat nordique, aux connaissances liées à l'agriculture nordique, aux compétences nécessaires tant techniques qu'administratives ainsi qu'à l'éloignement des grands centres. Cet éloignement fait en sorte que les spécialistes en agronomie, en santé animale, etc. sont difficilement accessibles. De plus, les commerces connexes (p. ex., les garages et les distributeurs de pièces pour les tracteurs) ne sont pas présents.

Lors des entretiens, deux initiatives en matière de production alimentaire ont été mentionnées, soit l'exploitation de la cueillette de champignons et la production en serre. Selon nos entretiens auprès de spécialistes en production alimentaire, la cueillette des champignons et autres produits forestiers non ligneux déjà présents dans la forêt pourrait être une 
activité économique importante. Toutefois, selon ces informateurs, une réflexion et un plan de développement sont nécessaires pour l'exploitation de cette ressource, pour sa rentabilité et pour les relations avec les Premières Nations, surtout si on adopte une perspective de développement durable.

\section{Les distributeurs}

Les distributeurs sont les gens qui travaillent à la vente de produits alimentaires. De manière générale, toutes les villes/localités de la Jamésie sauf une possèdent un point de vente de denrées alimentaires. Considérant la présence de ces commerces dans presque toutes les villes/localités de la Jamésie, les organismes communautaires qui réalisent des projets en sécurité alimentaire doivent être soucieux de l'impact qu'ils peuvent avoir sur le chiffre d'affaires de ces commerces. Certains intervenants ont tenté de mener des projets (p. ex., des jardins communautaires ou la vente de plants de légumes), mais quelques commerçants ont démontré un certain mécontentement. Pour les informateurs communautaires, le défi consiste en la complémentarité des services avec ces commerçants afin d'éviter de les concurrencer.

\section{Les transformateurs}

Aucune entreprise de transformation alimentaire n'a été mentionnée lors des entretiens. Certaines petites organisations vendent du thé du Labrador en sachet ou des champignons séchés. Des cuisines commerciales ont été identifiées dans les établissements d'hébergement, les restaurants, les centres de services hospitaliers, certaines épiceries et certaines grandes entreprises. Toutefois, l'accès ou le partage de ce genre d'installation n'a pas été signalé par les personnes participantes.

\section{Les transporteurs}

Pour ce qui est des transporteurs, différentes entreprises circulent sur le territoire, mais une seule est spécialisée dans les régions nordiques.

\subsection{Les acteurs publics et communautaires}

Des intervenants d'organismes communautaires ainsi que des professionnels œuvrant au sein des écoles primaires et des centres de formation professionnelle ont aussi participé aux entretiens.
Ces derniers, centrés sur les services alimentaires développés, portaient sur les besoins initiaux et sur les phases de développement des interventions réalisées.

Les trois écoles primaires et secondaires, qui affichent le plus haut indice de défavorisation ${ }^{5}$, offrent le service des soupes-repas aux élèves dans un réfrigérateur libre-service. Pour offrir ces aliments, les écoles ont comme partenaires des organismes communautaires. On peut donc observer une certaine cohérence entre les services alimentaires offerts et l'indice de défavorisation. Toutefois, un informateur rapporte que le succès de ce service dépend de la présence d'un organisme communautaire à proximité qui prend en charge sa gestion. Ce dernier précise que la mission des écoles est l'éducation et que celle des organismes communautaires peut être la sécurité alimentaire.

Le Portrait des services en sécurité alimentaire dans le Norddu-Québec, secteur Jamésie (TJSIS, 2016) confirme cette division des tâches et des missions. Selon ce portrait, $75 \%$ des interventions des organismes œuvrant en sécurité alimentaire consistent en dépannage (p. ex., repas communautaires, cartes d'achat en épicerie ou paniers de Noël), tandis que $25 \%$ des interventions visent l'autonomie alimentaire des personnes (p. ex., cuisines collectives, jardins communautaires ou services de soutien bénévole pour l'allaitement). Pour la plupart des personnes ayant participé aux entretiens, plusieurs activités privilégient la dimension de sociabilité pour briser l'isolement, bien au-delà du service alimentaire au sens strict.

\subsection{Les mangeurs}

Quelles sont les personnes les plus susceptibles de se retrouver en situation d'insécurité alimentaire? Il n'est pas facile de répondre à cette question, tant le portrait est hétéroclite. La faiblesse du revenu est évidemment mentionnée comme cause principale de l'insécurité alimentaire. Les informateurs font alors allusion aux personnes recevant des prestations de sécurité sociale, aux travailleurs à temps partiel et aux chômeurs.

Cependant, certains indiquent que même les travailleurs occupant un bon emploi à la mine ou en forêt peuvent avoir des difficultés à bien s'alimenter s'ils ont des problèmes de consommation de drogue ou d'alcool ou s'ils se révèlent incapables de 
bien gérer leur budget. Ayant été moins exposés à une situation de pauvreté, ils ont souvent de la difficulté à se débrouiller. La plupart des personnes interrogées estiment que l'incidence de la pauvreté financière, bien que réelle, reste très limitée dans la région.

La sécurité alimentaire est aussi influencée par les événements imprévisibles ou inévitables de la vie. Certaines personnes rencontrées ont fait part de leurs problèmes de santé et de ceux de leurs proches. Ces troubles peuvent avoir des conséquences sur le régime alimentaire et mener à des contraintes nutritionnelles. Les aliments nécessaires ne sont pas toujours disponibles dans les commerces locaux, car le marché pour ces produits est trop petit pour en assurer un inventaire permanent. Malgré une volonté parfois affirmée de privi- légier l'achat local, ces personnes doivent commander à l'extérieur et le transport peut altérer la qualité des produits.

D'autres événements peuvent influencer l'alimentation. Par exemple, un accident de travail (à soi ou au conjoint) ou une séparation affecte évidemment les revenus et peut obliger à recourir au dépannage, bien que ce ne soit pas une solution de rechange agréable.

Afin de combler leurs besoins alimentaires, les mangeurs proposent différentes stratégies, c'est-àdire un ensemble de moyens pris pour atteindre un objectif. En l'occurrence, cela signifie la façon dont les personnes s'y prennent pour atteindre une sécurité alimentaire. Comme nous le verrons, les participants font preuve de créativité; ils déploient en moyenne de 5 à 8 stratégies. Le tableau 1 présente des exemples de stratégies.

\begin{tabular}{|l|l|}
\hline Catégorie de stratégies & Exemples \\
\hline Personnelles & $\begin{array}{l}\text { Allaiter, diminuer les produits superflus, planifier les repas, récupérer des } \\
\text { denrées dans les poubelles, manger sur les lieux de travail }\end{array}$ \\
\hline \begin{tabular}{|l|l|} 
Par rapport aux achats en \\
épicerie
\end{tabular} & $\begin{array}{l}\text { Prendre un rendez-vous en même temps dans une autre ville qui a une } \\
\text { grande épicerie, faire son menu en fonction des rabais, faire une liste } \\
\text { d'épicerie, utiliser la livraison }\end{array}$ \\
\hline Dans la cuisine & $\begin{array}{l}\text { Cuisiner tous les repas, cuisiner en groupe, partager des recettes, congeler } \\
\text { ou stocker des denrées }\end{array}$ \\
\hline De production alimentaire & $\begin{array}{l}\text { Chasser, pêcher, cueillir des fruits sauvages, se faire un jardin privé ou une } \\
\text { serre maison, avoir des poules pondeuses }\end{array}$ \\
\hline \hline $\begin{array}{l}\text { Entre individus, d'entraide } \\
\text { Avec les milieux } \\
\text { communautaire et public }\end{array}$ & $\begin{array}{l}\text { Covoiturer, acheter pour ou par d'autres les denrées en rabais, emprunter } \\
\text { de l'argent, groupe d'achats, partager/donner, troquer }\end{array}$ \\
\hline \hline & $\begin{array}{l}\text { Utiliser les services de la popote roulante, du dépannage alimentaire, des } \\
\text { repas communautaires, de la cuisine collective, du jardin communautaire, } \\
\text { d'aide à domicile, de la soupe dans un réfrigérateur libre-service }\end{array}$ \\
\hline
\end{tabular}

Tableau 1 - Exemples de stratégies alimentaires déployées

Les 24 informateurs «mangeurs» mentionnent déployer annuellement entre 5 et 8 stratégies différentes pour s'alimenter. Les stratégies ne diffèrent pas de façon marquée d'une ville/localité à l'autre. C'est plutôt la présence d'enfants dans les ménages qui fait varier à la hausse le nombre de stratégies.

Globalement, les personnes participant à la recherche semblent maitriser différentes compétences telles que cuisiner, planifier des achats, jardiner, dépecer un animal, pêcher, cueillir, etc. Elles possèdent de l'espace et plusieurs équipements et accessoires liés à la cuisine, à la congélation, à la pêche, à la chasse et au jardinage en terre ou en serre, sans oublier une voiture pour se rendre à l'organisme communautaire ou à l'épicerie à l'extérieur de la ville. De plus, ces personnes ont bâti un réseau de soutien avec des membres de leur famille pour faire des achats, prêter de l'argent, se déplacer en voiture, ou encore avec des organismes communautaires et publics (p. ex., l'école). 


\section{Discussion}

Dans ces petites villes/localités où tous les gens se connaissent, se côtoient régulièrement et partagent des défis similaires pour s'alimenter, les stratégies alimentaires commencent à se diversifier, à se multiplier et à mobiliser de plus en plus de personnes. De plus, puisqu'il s'agit de petites localités éloignées les unes et des autres, l'entraide entre les commerces et envers les mangeurs est un facteur essentiel de genèse des innovations.

Les données montrent que les acteurs (incluant les mangeurs) ont développé au cours du temps diverses stratégies pour favoriser leur sécurité alimentaire et/ou pour éviter l'insécurité alimentaire. Ces stratégies révèlent l'importance des compétences et des ressources personnelles ainsi que des réseaux interpersonnels (famille, amis et voisins). Les gens n'agissent pas seuls, mais au sein d'un univers interactionnel. On aurait donc tort d'approcher la résilience des acteurs comme une caractéristique purement individuelle : elle se manifeste aussi (ou se déploie et se réalise) au moyen d'«actes réticulaires », c'est-à-dire par l'activation de réseaux et par la création de collectifs, pour reprendre le terme de Latour (2007).

La présence du secteur associatif, qui concerne les organismes communautaires, montre que ces stratégies demandent une « infrastructure sociale», autre preuve qu'une approche strictement individualiste ne convient pas. Il y a une boucle de rétroaction (feedback loop), c'est-à-dire une interdépendance des acteurs et de leurs actions : les actions individuelles profitent de la présence de ces « infrastructures» (de ce point de vue, celles-ci précèdent les actions), mais elles sont aussi l'occasion de leur mise sur pied (de ce point de vue, les actions individuelles précèdent les infrastructures). Nous ne sommes pas face à une causalité linéaire, mais à une relation systémique. De plus, comme on le sait, ces stratégies sont égocentrées, mais elles fonctionnent à la réciprocité et au lien social. Elles ne sont pas marchandes, même si elles interagissent évidemment avec des institutions marchandes (commerces, etc.).

En résumé, comme le rappellent Les Greniers d'Abondance (2020), il est nécessaire de travailler à l'aide d'une approche systémique impliquant les acteurs du système alimentaire parce qu'une menace peut entraîner des répercussions graves à l'ensemble. Cette approche est adaptée pour renforcer la cohérence des actions des acteurs publics et communautaires en matière de résilience alimentaire.

Les acteurs interrogés font une analyse parfois poussée de leurs stratégies et tentent de les améliorer. Cette analyse inclut la mobilisation de diverses compétences nécessaires pour réaliser les stratégies (p. ex., cuisine, planification des achats, culture maraîchère, dépeçage d'un animal ou d'un poisson), mais aussi la mobilisation d'équipements (cuisine, chasse/pêche, jardin/serre, congélateur, voiture) et d'espace (dans la maison ou sur le terrain extérieur). Les participants ajoutent que la variété de leurs stratégies est possible lorsqu'ils ne vivent aucun événement imprévu (p. ex., maladie, accident ou pandémie). Ils précisent que leurs ressources financières leur permettent d'être plus autonomes et facilitent la réalisation de leurs actions alimentaires. Selon le continuum des capacités en matière de résilience de Béné et ses collègues (2016), cette analyse peut démontrer une certaine capacité de résistance des ménages pour éviter l'insécurité alimentaire, bien qu'elle reste fragile.

En effet, cette capacité peut être mise à rude épreuve lors d'événements imprévus. Afin de répondre aux besoins alimentaires ponctuels de ces mangeurs, de nombreuses associations peuvent offrir gratuitement des denrées, ce qui est conforme au modèle de Béné et ses collègues (2016) et de la FAO (2008). Les acteurs économiques et communautaires proposent des activités de manière individuelle et développent des stratégies pour les améliorer, mais sans nécessairement partager leurs expériences. Il en résulte que plusieurs répètent les erreurs ou remplissent les mêmes documents que d'autres avant eux. À cet égard, ces acteurs du système alimentaire pourraient être davantage perçus dans un état de persistance (Béné et collab., 2016) en démontrant une capacité à supporter les perturbations extérieures. Un dialogue entre ces acteurs leur permettrait de capitaliser leurs réussites et celles des autres pour favoriser le développement d'une capacité adaptative ou même transformatrice de leur résilience.

Toutefois, les acteurs du système alimentaire soulignent que leur milieu présente des défis en matière de développement, en particulier si on considère 
l'interdépendance nécessaire avec d'autres acteurs économiques, publics, communautaires ou avec des mangeurs. L'isolement géographique semble contraindre tous ces acteurs à penser à des idées ou à des innovations sociales plus collectives.

Pour les acteurs économiques, la coopération entre entrepreneurs ne semble pas aussi facile que pour les autres types d'acteurs, même si ceux-ci donnent l'exemple du service des soupes-repas dans certaines écoles par les organismes communautaires. La mutualisation des équipements et des connaissances pourrait être un point de ralliement pour des acteurs partageant les particularités nordiques de leur région. Plus encore, une localité qui possède une infrastructure imposante pourrait devenir un pôle de référence pour les acteurs préoccupés par la production alimentaire. Le partage de l'information, des stratégies alimentaires et des compétences de tous les acteurs pourrait être un angle de développement du secteur agroalimentaire tout autant que des communautés.

D'ailleurs, l'absence de concertation entre les acteurs du système alimentaire explique probablement que le niveau actuel de résilience du système alimentaire est bas. Toutefois, les actions et les réflexions livrées par les participants suggèrent un réel potentiel dynamique d'amélioration de la résilience alimentaire des différents maillons du système alimentaire.

Enfin, puisque l'insécurité alimentaire est vécue par une grande diversité de personnes, il serait pertinent d'encourager l'émergence de stratégies des acteurs qui incluent tous les mangeurs, peu importe leur situation. Cette proposition permet de préserver la dignité des mangeurs puisque, dans les petites villes et localités, l'anonymat semble impossible. Pour renforcer cette capacité adaptative de la résilience alimentaire, les acteurs subventionnaires pourraient apporter des améliorations structurelles et durables, une opinion reprise par Fallot et ses collègues (2019), pour qui cette vision administrative de la résilience alimentaire est soutenue par une canalisation des actions vers une sécurité alimentaire souhaitable. Le tableau 2 résume les obstacles et les facteurs facilitants en matière de stratégies innovantes.

\begin{tabular}{|l|l|}
\hline Obstacles & Facteurs facilitants \\
\hline Difficultés de transport, distance & Présence d'acteurs stratégiques \\
\hline Compétences techniques, connaissances & Contexte organisationnel \\
\hline \hline Climat & Programmes gouvernementaux \\
\hline $\begin{array}{l}\text { Événements stresseurs imprévus (séparation, perte } \\
\text { d'emploi, maladie, pandémie, etc.) }\end{array}$ & Concertation entre les acteurs du système alimentaire \\
\hline
\end{tabular}

Tableau 2-Obstacles et facteurs facilitants en matière de stratégies innovantes

\section{Conclusion}

Ce texte a présenté certains des résultats d'une recherche sur les stratégies déployées par des acteurs du système alimentaire de la Jamésie, une région nordique du Québec. Leur contexte est marqué par un climat rigoureux, par un éloignement des marchés et par la distance entre les collectivités, mais aussi par la proximité sociale découlant de la petite taille des villes et localités, par un fort tissu associatif local et par des modes d'action privilégiant les partenariats entre services publics et organisations communautaires locales.
L'interdépendance entre les secteurs du système et l'importance d'imaginer des voies innovantes à la recherche de sécurité alimentaire sont certainement des traits marquants de la situation de ces villes et localités. Or, la nécessité de coordonner les actions, leur intégration à des politiques publiques faisant une plus large place aux administrations locales et une mobilisation accrue des ressources citoyennes le sont tout autant. En d'autres termes, les actions doivent s'appuyer sur une vision de développement.

Cette vision de développement que doivent supporter les politiques publiques devrait inclure une 
définition de la sécurité alimentaire pour tous. Dans cet outil de coordination visant le bien commun, la notion de sécurité alimentaire peut comporter un large éventail de significations sans se limiter à la production locale et peut être assez éloignée de l'autonomie alimentaire.

Les régions comme la Jamésie ne produisent pas et ne pourront sans doute jamais produire tous les aliments dont elles ont besoin. En effet, la saison propice à la culture est très courte, ce qui réduit les possibilités agricoles. La culture en serre, une façon de contourner les difficultés du climat, a des exigences technologiques et énergétiques coûteuses qui demandent des compétences particulières. Le réchauffement climatique modifiera sûrement ces conditions, bien qu'il soit difficile de savoir exactement de quelle manière, mais on peut penser que les cultures potagères seront plus aisées. Reste à voir si la culture locale et, plus généralement, le mode de vie d'une région marquée par l'économie extractive sauront profiter de ces changements.

D’ici là, la région jamésienne devra continuer à utiliser des aliments provenant d'ailleurs. La sécurité alimentaire de sa population demande donc qu'on lui assure l'accès aux aliments nécessaires, ce qui implique de considérer les exigences du transport, les coûts associés et les contraintes de conservation des aliments.

Dans le cadre de cette vision de développement, la résilience d'un système alimentaire ne peut être pensée sans considérer les comportements orientés - les stratégies - des acteurs de ce système. On peut les concevoir comme visant fondamentalement la satisfaction des besoins des personnes, qui peuvent en être conscientes ou non, mais cette détermination est loin de couvrir l'entièreté des possibles, ne serait-ce que parce que la notion de besoin n'a rien d'évident ni de simple.

La mise sur pied de ces stratégies demandera une planification opérationnelle des moyens, qui peuvent être matériels, techniques, informationnels, organisationnels ou sociaux, ou encore impliquer d'autres acteurs ayant eux aussi leurs stratégies. Le réseau que forme l'entrecroisement de ces actions est complexe et peut se déployer à plusieurs échelles territoriales. Nous pouvons émettre l'hypothèse que la capacité du système à se maintenir, soit sa résilience, sera d'autant plus forte que ce réseau aura de redondance (plusieurs moyens concurrents pour arriver à une fin comparable) et saura générer des innovations afin de s'adapter aux changements de l'environnement du système et/ou de le modifier. La distance est un des principaux facteurs contraignants de la sécurité alimentaire. Elle a aussi des conséquences évidentes sur les revenus nécessaires à une saine alimentation.

En somme, il ressort de cet article que la résilience alimentaire exige une multiplicité de stratégies tant individuelles que collectives nécessitant une orchestration efficace et efficiente. Cette orchestration peut se faire par l'adoption d'une politique publique favorable à la sécurité alimentaire pour tous les citoyens et citoyennes avec tous les acteurs du système alimentaire. Afin de maximiser ces efforts, un partenariat avec les Premières Nations qui habitent également ce territoire devrait être envisagé.

\section{Limites}

Cet article n'a fait qu'effleurer une question bien plus vaste. D'autres recherches devraient insister sur la résilience alimentaire des Premières Nations ainsi que sur les liens à développer avec elles, car nous n'avons pas abordé les relations entre les allochtones et la Première Nation crie, malgré l'expertise que celle-ci a développée. Une certaine priorisation des stratégies pourrait être également étudiée afin de maximiser les efforts des acteurs impliqués.

De même, la préoccupation liée à l'accès universel à l'alimentation devrait inclure une discussion relative à l'empowerment et à la résilience.

Enfin, les régions similaires à la Jamésie devraient faire l'objet de recherches plus nombreuses, car elles obligent à une définition plus vaste de la sécurité alimentaire, trop souvent ramenée à la production locale et aux circuits courts. Que faire alors des endroits où ceux-ci sont impossibles? 


\section{NOTES}

1 La recherche est celle de Desjardins et Tremblay (2021).

2 «Petite ville, village ou toute agglomération plus importante que le hameau non nécessairement incorporé comme une entité administrative distincte.» (Grand dictionnaire terminologique)

3 Pour Mulgan (2006), une innovation sociale est une activité ou un service répondant à un besoin social. Il s'agit d'un changement ou d'une rupture avec la situation actuelle. Le point de départ de l'innovation débute par l'identification d'un besoin non satisfait par un groupe d'individus qui ont des idées pour le combler. Ces idées issues d'un bricolage d'expériences passées et de suggestions sont déployées, pour être ensuite évaluées et diffusées, donc communiquées.

4 Compilation réalisée par les auteurs à partir des documents du ministère du Développement durable, de l'Environnement et des Parcs ainsi que des portraits réalisés par des instances locales de la Baie-James.

5 Voir la définition de l'indice de défavorisation matérielle et sociale sur le site de l'Institut national de santé publique du Québec: https://www.inspq.qc.ca/defavorisation/indice-de-defavorisation-materielle-et-sociale

\section{RÉFÉRENCES}

Banques alimentaires du Québec (BAQ). (2020). Rapport d'étape COVID-19, de mars à juin 2020.

Béné, C., Headey, D., Haddad, L. et von Grebmer, K. (2016). Is resilience a useful concept in the context of food security and nutrition programmes? Some conceptual and practical considerations. Food Security, 8(1), 123-138. https://doi.org/10.1007/s12571-015-0526-x

Bergeron, O., Richer, F., Bruneau, S. et Laberge-Gaudin, V. (2015). L'alimentation des Premières Nations et des Inuits au Québec. Institut national de santé publique du Québec. https://www.inspq.qc.ca/pdf/publications/2054_alimentation_premieres_ nations_inuits.pdf

Centre régional de la santé et des services sociaux (CRSSS) de la Baie-James. (2020). Rapport annuel de gestion 2019-2020. Gouvernement du Québec. http://www.crsssbaiejames.gouv.qc.ca/1341/Publications.crsssbaiejames

Côté, J. (2007). Le quotidien de la pawreté et de l'insécurité alimentaire en Gaspésie-Îles-de-la-Madeleine [rapport de recherche]. Agence de santé et des services sociaux Gaspésie-Îles-de-la-Madeleine. https://numerique.banq.qc.ca/patrimoine/ details/52327/459203?docref=yJZHAMnrT8Rp4UW5B868NA

Creswell, J. W. et Poth, C. N. (2018). Qualitative inquiry and research design: Choosing among five approaches (4e éd.). SAGE.

Damon, J. (2012). La question SDF. PUF.

Department for International Development (DFID). (2011). Defining disaster resilience: A DFID approach paper. Department for International Development/UKaid. https://www.fsnnetwork.org/sites/default/files/dfid_defining_disaster_resilience.pdf

Desjardins, F. et Tremblay, P.-A. (2021). La sécurité alimentaire en Jamésie : rapport final de la recherche. Université du Québec à Chicoutimi, Groupe de recherche et d'intervention régionales. https://www.academia.edu/45093496/La_s\%0C3\%A9curit\%C3\%A9_ alimentaire_en_Jam $\%$ C3\%A9sie_Rapport_final_de_la_recherche_remis_au_R\%C3\%A9seau_jam\%C3\%A9sien_ de_d $\%$ C $3 \%$ A9veloppement_social

Dupéré, S., Gélineau, L., Adam, G., Côté, M., Dufour, É., Dumas, A., ... et Bélisle, M. (2014). Vers une autonomie alimentaire pour toutes: Agir et Vivre Ensemble le Changement (AVEC). Rapport de recherche. https://frq.gouv.qc.ca/app/uploads/2021/08/pauvrete2011-2012_dupere_s_rapport-final.pdf

Fallot, A., Bousquet, F. et Dury, S. (2019). Les paradoxes de la résilience en matière de sécurité alimentaire. Revue internationale des études du développement, 3(239), 57-87. https://doi.org/10.3917/ried.239.0057

Folke, C., Carpenter, S. R., Walker, B., Scheffer, M., Chapin, T. et Rockström, J. (2010). Resilience thinking: Integrating resilience, adaptability and transformability. Ecology and Society, 15(4), 20. http://www.ecologyandsociety.org/vol15/iss4/art20

Latour, B. (2007). Changer de société, refaire de la sociologie. La Découverte.

Latour, B. (2015). Face à Gä̈a : buit conférences sur le nouveau régime climatique. La Découverte.

Les Greniers d'Abondance (2020) Vers la résilience alimentaire. Faire face aux menaces globales à l'échelle territoriale. Première édition. https://resiliencealimentaire.org/

Malassis, L. (1994). Nourrir les hommes. Flammarion. 
Mélèze, J. (1972). L'analyse modulaire des systèmes de gestion : une méthode efficace pour appliquer la théorie des systèmes au management. Éd. Hommes et techniques.

Miles, M. B. et Huberman, A. M. (1994). Qualitative data analysis (2e éd.). SAGE.

Mulgan, G. (2006). The process of social innovation. Innovations: Technology, governance, globalization, 1(2), 145-162. http://www.mitpressjournals.org/doi/pdf/10.1162/itgg.2006.1.2.145

Organisation des Nations unies pour l'alimentation et l'agriculture (FAO). (1996). Déclaration de Rome sur la sécurité alimentaire mondiale. https://www.fao.org/3/W3613F/W3613F00.htm

Organisation des Nations unies pour l'alimentation et l'agriculture (FAO). (2008). Sécurité alimentaire : l'information pour l'action - Guides pratiques. https://www.fao.org/3/al936F/al936F.pdf

Pageau, M. (dir.). (2008). Cadre de référence en matière de sécurité alimentaire : mise à jour 2008. Direction des communications, ministère de la Santé et des Services sociaux du Québec. https://publications.msss.gouv.qc.ca/msss/fichiers/2008/08-208-01.pdf

Québec. Loi visant à lutter contre la pawreté et l'exclusion sociale. RLRQ, chap. L-7, à jour au 1er août 2021, [Québec], Éditeur officiel du Québec, 2002. http://legisquebec.gouv.qc.ca/fr/ShowDoc/cs/L-7

Rastoin, J.-L. et Ghersi, G. (2010). Le système alimentaire mondial : concepts et méthodes, analyses et dynamiques. Éditions Quae.

Statistique Canada. (2016). Recensement de 2016. Gouvernement du Canada. https:/ /www12.statcan.gc.ca/ census-recensement/2016/dp-pd/index-fra.cfm

Statistique Canada. (2017). Enquête sur la santé dans les collectivités canadiennes (ESCC) 2017. Gouvernement du Canada. https://www23.statcan.gc.ca/imdb/p2SV_f.pl?Function=getSurvey\&Id=329241

Statistique Canada. (2018). Enquête sur la santé dans les collectivités canadiennes (ESCC) 2018. Gouvernement du Canada. https://www23.statcan.gc.ca/imdb/p2SV_f.pl?Function=getSurvey\&Id=795204

Table jamésienne pour la solidarité et l'inclusion sociale (TJSIS). (2016). Portrait des services en sécurité alimentaire dans le Nord-du-Québec, secteur Jamésie. Centre régional de santé et de services sociaux de la Baie-James.

Tendall, D. M., Joerin, J., Kopainsky, B., Edwards, P., Shreck, A., Le, Q. B., Kruetli, P., Grant, M. et Six, J. (2015). Food system resilience: Defining the concept. Global Food Security, 6, 17-23. https://doi.org/10.1016/j.gfs.2015.08.001

Touzard, J.-M. et Fournier, S. (2014). La complexité des systèmes alimentaires : un atout pour la sécurité alimentaire? VertigO, 14(1). https://doi.org/10.4000/vertigo.14840

United Nations Fund for Population Activities (UNFPA). (2015). State of world population 2015: Shelter from the storm. A transformative agenda for women and girls in a crisis-prone world. United Nations Population Fund. https://www.unfpa.org/sites/ default/files/sowp/downloads/State_of_World_Population_2015_EN.pdf

United States Agency for International Development (USAID). (2012). Building resilience to recurrent crisis - US AID policy and program guidance. U.S. Agency for International Development. https://www.usaid.gov/sites/default/files/documents/1866/ Policy $\% 20 \% 26 \% 20$ Program $\% 20$ Guidance $\% 20-\% 20$ Building\%20Resilience $\% 20$ to\%20Recurrent $\% 20$ Crisis_Dec $\% 202012$.pdf

Vonthron, S., Dury, S., Fallot, A., Alpha, A. et Bousquet, F. (2016). L’intégration des concepts de résilience dans le domaine de la sécurité alimentaire : regards croisés. Cabiers Agricultures, 25(6), 1-8. https://doi.org/10.1051/cagri/2016039 